\title{
Removal of embolised catheters using flexible endoscopy forceps
}

\author{
T R D SHAW \\ From the Department of Cardiology, Western General Hospital, Edinburgh
}

SUMMARY Five cases are described in which embolised catheters were removed percutaneously from the right side of the heart. Flexible endoscopy biopsy forceps were used in four cases and achieved removal in two: in the two other cases the catheter lay in a position which could not be reached by the biopsy forceps mouth, and retrieval was made with a helical wire basket after dislodgment of the proximal end of the catheter. In a fifth case a catheter fragment was removed from the pulmonary artery with endoscopy grasping forceps. Flexible endoscopy forceps provide a very firm grip and with a Teflon sleeve they can be negotiated around curves. They have limited manoeuvrability but W-shape "grasping" forceps have a large mouth, which helps to overcome this limitation.

Central venous cannulation is increasingly used for parenteral feeding and cancer chemotherapy. Occasionally the catheter may sever or break from its connector and embolise to the right side of the heart. ${ }^{1}$ Five cases are described in which attempts were made to remove embolised catheters using flexible forceps designed for fibreoptic endoscopy.

\section{Case reports}

\section{CASE 1}

A 17 year old male patient with leukaemia had been receiving chemotherapy via a Vygon "Nutricath S" silicone catheter inserted at the right subclavian vein. The catheter separated from its proximal connector. A chest $x$-ray film showed the proximal end to be in the subclavian vein adjacent to the medial head of the clavicle and the distal end to be against the inferior wall of the right ventricle.

An Olympus gastroscopy biopsy forceps, sterilised by glutaraldehyde (Cidex), was advanced via an F9 Vygon introducer from the right femoral vein to the right atrium. Control of opening and closing of the mouth of this type of forceps is by a push and pull handle. When the tip of the forceps was in the right atrium the mouth was opened. It advanced past the catheter but did not engage it, indicating that catheter and forceps were not in the same vertical plane. With

Accepted for publication 6 July 1982 the patient rotated from the supine to the $30^{\circ}$ right anterior oblique position, however, the mouth of the forceps caught the catheter as it lay within the midatrium (Fig. 1a). Gravity had altered the plane of the forceps sufficiently to bring it into line with the catheter. The mouth of the forceps was closed and the catheter withdrawn to the femoral vein. The introducer was removed and the catheter was pulled through the skin.

CASE 2

A 60 year old man had a Nutricath catheter in the right subclavian vein for parenteral feeding after bowel resection for carcinoma. The catheter separated from its connector and embolised to the same position within the right side of the heart as in case 1 .

The biopsy forceps were positioned in the right atrium in the manner described above. The forceps, however, could not be made to engage the catheter as it crossed the right atrium, even when the patient was turned into the oblique position. The forceps were advanced to the superior vena cava. In this area the catheter made a loop towards the midline. The forceps grasped the catheter as it moved medially in systole (Fig. 1b). It could be seen that the forceps had grasped only part of the diameter of the catheter. Firm pressure was maintained on the forceps handle while the catheter was withdrawn to the femoral vein and then through the skin. One edge of the mouth of the forceps had partially bitten through the catheter but good contact had been maintained at the other edge. 


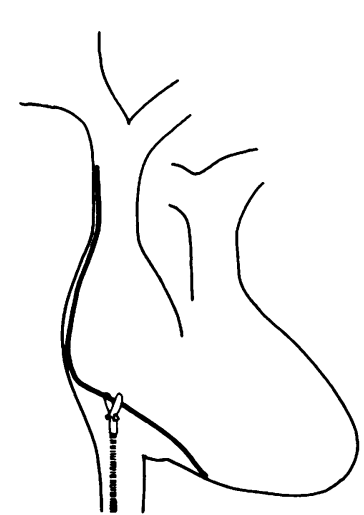

a

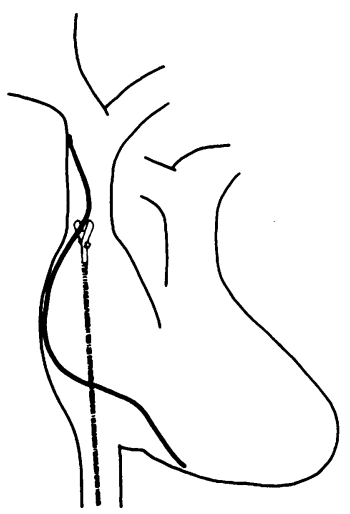

b

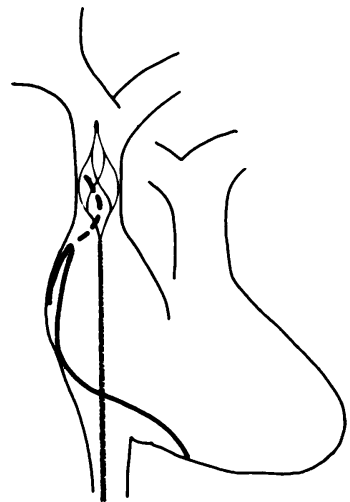

C

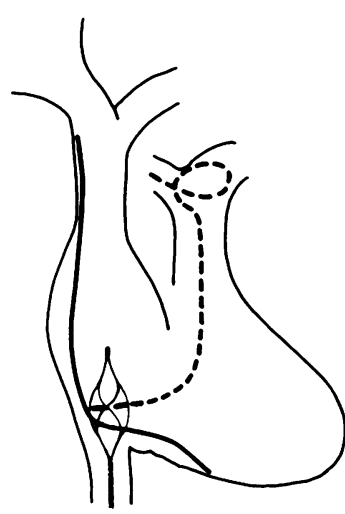

d

Fig. la-d Position of the embolised catheters in cases 1 to 5 , and the methods of retrieval. The solid line indicates the intitial position of the catheter: the dashed line shows the position after dislodgement (see text).

\section{CASE 3}

A woman, aged 70 , received parenteral feeding via a Nutricath catheter after operation for a ruptured duodenal diverticulum complicated by wound infection and a fistula. After insertion into the right subclavian vein the infusion line was noted to be leaking and a $20 \mathrm{~cm}$ portion of the catheter was found to be missing, apparently severed by a stitch. A chest $x$-ray film showed the missing portion to be within the right atrium and ventricle. She was transferred for removal of the catheter four weeks after its initial embolisation.

Fluoroscopy showed that the proximal portion of the catheter fragment was curved against the upper wall of the right atrium while the distal portion was within the right ventricle (Fig. 1c). Forceps were passed to the right atrium but did not engage the catheter as it crossed the atrial cavity. The proximal end of the catheter curved within the atrium and lay too far laterally to be caught by the forceps at the upper atrial level. A Dotter retrieval basket set (Cordis Inc.) was inserted. It did not catch the catheter at initial attempts but finally dislodged the proximal end which then floated into the superior vena cava. After withdrawal of the basket down the superior vena cava the free floating end was ensnared (Fig. 1c). The basket was closed and the catheter withdrawn through the skin.

\section{CASE 4}

A 44 year old woman had resection of large bowel for Crohn's disease and was receiving intravenous fluids via a Nutricath catheter inserted into the right internal jugular vein. The catheter became blocked and was flushed with $5 \mathrm{ml}$ saline. The catheter became detached from its connector and was seen on $x$-ray film to have its proximal tip at the lower end of the jugular vein and its distal tip near the tricuspid valve.

Three days later the patient was referred for catheter removal. By this time the catheter had moved and its proximal end was now at the middle of the superior vena cava and the distal end was within the right ventricle (Fig. 1d). Bronchoscopy flexible biopsy forceps were inserted into the right femoral vein but met resistance at the lower end of the inferior vena cava. A Teflon guidewire and sleeve were passed to the right atrium and the forceps were then easily negotiated to the right atrium via the sleeve. The forceps failed to grasp the catheter within the right atrium. When advanced to the superior vena cava the forceps lay close to the catheter, but the absence of any loop on the catheter, even during deep inspiration, meant that the catheter and forceps lay parallel and the mouth of the forceps did not engage the catheter.

The basket catheter was inserted via the femoral vein and passed up to the upper superior vena cava. It was then opened and drawn slowly downwards. The catheter was not caught but suddenly moved and its distal end was seen to be coiled in the main pulmonary artery. The basket was brought to the right atrium, and then closed. On withdrawal the catheter followed and after removal was found to have been snared by the basket $1 \mathrm{~cm}$ from its proximal end.

These cases showed that limited manoeuvrability made the biopsy forceps unsuccessful when the catheter did not lie close to the line of the forceps. They also illustrated the disadvantage of the basket retrieval set, namely that partial dislodgment of the catheter was needed when no free end was available and this could cause further embolisation. It seemed likely that positioning of the biopsy forceps could be improved by passing them through a preshaped large 
bore cardiac catheter, but a more attractive possibility was found in one of the other types of flexible forceps in the Olympus range-the W-shape grasping forceps which had a much larger mouth than the biopsy model (Fig. 2). An opportunity to use this type of forceps arose with the next case.

\section{CASE 5}

A 43 year old male patient was noted at a cardiac follow-up clinic to have a fragment of catheter visible in the right upper zone of his chest $x$-ray film. He had had coronary artery graft surgery 18 months earlier. The fragment had not been seen on earlier films and had presumably been within the cardiac silhouette after embolising at the perioperative period.

On fluoroscopy one end of the catheter lay in an upper branch of the right pulmonary artery, with the tip $1.5 \mathrm{~cm}$ below the lower edge of the clavicle. The proximal end was within the main right pulmonary artery (Fig. 3). A guidewire was inserted via the right femoral vein and positioned in a distal right pulmonary artery branch. A long sheath introducer set (Cook Inc.) was passed over the wire to this distal site. The wire and guiding catheter were withdrawn, leaving the long Teflon sleeve in position. The W-shape grasping forceps were introduced into the sleeve and passed easily around the curves to reach the end of the right pulmonary artery. The sleeve was partially withdrawn and the mouth of the forceps opened. The open forceps were passed back and forward near the catheter as it lay at the end of the right pulmonary artery, with different positions of rotation being used each time. The catheter fragment was seen to be moved slightly by the mouth and the grasping forceps were closed and the fragment withdrawn back and though the skin. Modest resistance was felt as the
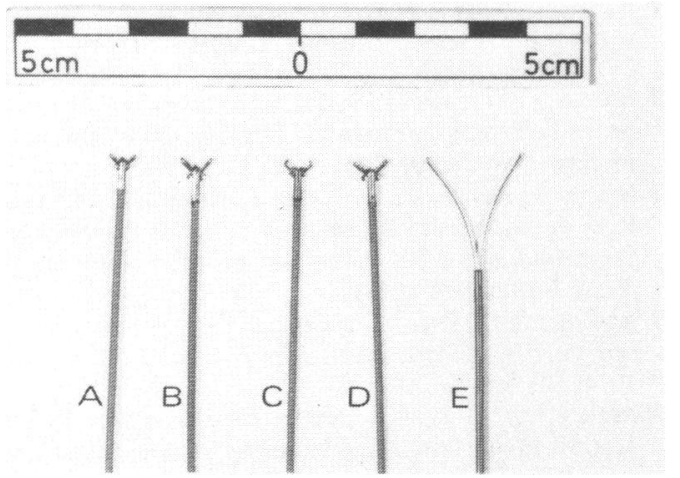

Fig. 2 Five types of flexible endoscopy forceps. A: standard biopsy forceps. B: Biopsy forceps with ellipsoid mouth. $C$ : Grasping forceps with "rat-tooth" mouth. D: Grasping forceps with "alligator" mouth. E: Grasping forceps with "W-shape" mouth. "W-shape" refers to the configuration of the small teeth at the end of each half of the forceps mouth.

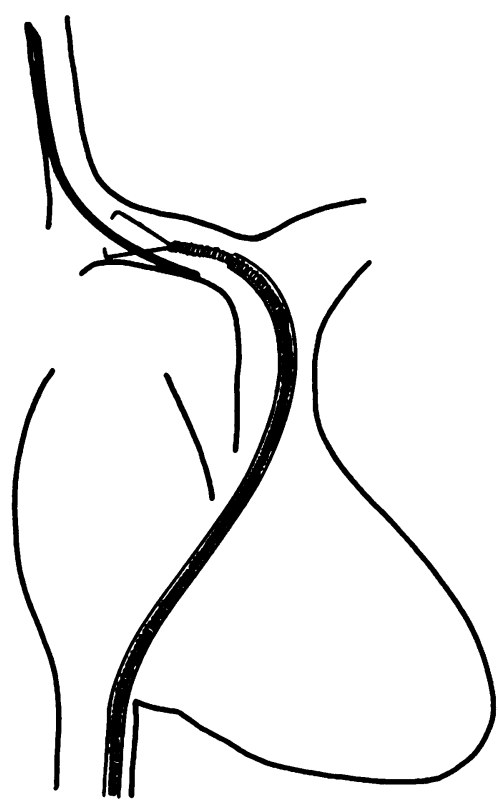

Fig. 3 Position of the embolised fragment in case 5. The W-shape grasping forceps have been passed to the right pulmonary artery via a Teflon sleeve (see text).

fragment was near the pulmonary valve: after removal, examination of the $8 \mathrm{~cm}$ fragment, now pulled into a V shape, showed it had become hardened during its time within the circulation and this probably accounted for the slight resistance as it came back across the pulmonary valve.

\section{Discussion}

Removal of embolised catheters is almost always indicated since without removal there is a very high incidence of death or serious morbidity caused by perforation of the heart, sepsis, pulmonary emboli, and arrhythmias. ${ }^{2-4}$

When part of an embolised catheter remains in an accessible vein a cutdown is the preferred method of retrieval. If this is not feasible, then non-surgical retrieval should be considered before resorting to thoracotomy. A variety of non-surgical methods has been used for removal of embolised material within the circulation. The techniques have been reviewed by Fisher and Ferreyro ${ }^{2}$ and by Bloomfield. ${ }^{5}$

If one end of the catheter is free floating, it may be captured with a loop snare made from a doubled-over guidewire passed through a cardiac catheter. A loop can also be made by tying a suture to the flexible end of a guidewire. A helical wire basket system, similar to that used for removal of ureteric stones, is also suitable when a free end is present. A loop snare sys- 
tem and a helical basket for cardiac use are marketed (Cook Inc.).

When there is no free floating end, retrieval is more difficult. It may be possible to remove the embolised fragment using a cardiac catheter or guidewire, with the distal end formed into a hook, but a combination of techniques is usually needed, with a hook device being used to detach one end of the catheter which is then able to be snared. ${ }^{6}$ This carries the risk of further embolisation after detachment. An alternative approach is to grasp the embolised catheter with forceps. Rigid bronchoscopy forceps have been successfully used to remove catheters from the right side of the heart. ${ }^{347}$ These forceps were inserted via a cutdown to the saphenous or jugular veins. They had the disadvantage of short length and their rigidity increased the risk of perforation and limited their manoeuvrability. 5 Endomyocardial bioptomes are flexible and this reduces these disadvantages. They have been used to remove foreign bodies from the right atrium and great veins. ${ }^{8-10}$ These instruments, however, are not widely available.

Flexible forceps for gastrointestinal endoscopy and bronchoscopy are now used in many hospitals and will often be readily available for urgent removal of embolised objects. They can grasp soft catheters very firmly: in vitro testing showed that the standard biopsy forceps held a catheter tied to weights up to $1 \cdot 1 \mathrm{~kg}$. Forceps for endoscopy (Olympus Optical Co Ltd: UK-KeyMed Ltd) are designed to fit channels of 2.0 or $2.8 \mathrm{~mm}$. The smaller size can be inserted via introducers of F6 size. Lengths of 100 to $185 \mathrm{~cm}$ are available. The forceps set, including the plastic handle, can be sterilised by immersion in cold solution or by ethylene oxide.

In adult cases a large introducer can be used initially to create a looser exit point at the skin. With more rigid embolised fragments insertion via a cutdown might be preferable. Even without heparinisation these forceps showed very little tendency to develop any clot on their surface. The forceps passed easily through a Teflon sleeve. This allows passage past any kinking of the veins and permits the forceps to be passed to the pulmonary artery.

The disadvantage of these forceps is their limited manoeuvrability. In case 1 the catheter lay close to the forceps and could be grasped within the atrium after repositioning of the patient. In the second case a curve in the alignment of the catheter in the superior vena cava allowed contact with the forceps mouth. Curvature can be accentuated by inspiration and increases during systole. The forceps were unsuccessful in two cases in which the forceps did not engage the catheter within the atrium and where there was no curvature towards the midline in the superior vena cava. In both instances the basket retrieval set was successful, but case 4 illustrates the danger of further embolism which can occur with techniques that dislodge part of the catheter. The W-shape flexible endoscopy "grasping" forceps have a much wider mouth and appear attractive for intravascular retrieval. Fig. 2 shows five types of flexible forceps, including the ellipsoid biopsy type used in the first four cases described here, and the W-shaped grasping forceps used in case 5. These grasping forceps overcome much of the difficulty of limited manoeuvrability.

In an individual case the method of retrieval has to be based on the position and nature of the embolised fragment. A variety of approaches may have to be tried. Flexible forceps are one option for selected cases.

\section{References}

1 Henzel JH, DeWeese MS. Morbid and mortal complications associated with prolonged central venous cannulation. Am F Surg 1971; 121: 600-5.

2 Fisher RG, Ferreyro R. Evaluation of current techniques for nonsurgical removal of intravascular iatrogenic foreign bodies. $A \mathcal{F}$ 1978; 130: 541-8.

3 Dotter CT, Rösch J, Bilbao MK. Transluminal extraction of catheter and guide fragments from the heart and

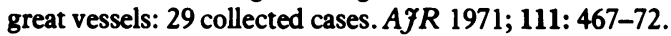

4 Bloomfield DA. Techniques of nonsurgical removal of iatrogenic foreign bodies from the heart. Am $\mathcal{f}$ Cardiol 1971; 27: 538-45.

5 Bloomfield DA. The nonsurgical retrieval of intracardiac foreign bodies - an international survey. Cathet Cardiovasc Diagn 1978; 4: 1-14.

6 Rossi P, Passariello R, Simonetti G. Intravascular iatrogenic foreign body retrieval. Ann Radiol (Paris) 1980; 23: 286-90.

7 Smyth NPD, Rogers JB. Transvenous removal of catheter emboli from the heart and great vessels by endoscopic forceps. Ann Thorac Surg 1971; 11: 403-8.

8 Kurita A, Kanazawa M, Kanie T, Kimura E, Nakayama R, Shoji T. Successful removal of a foreign body from the caval vein by use of endo-myocardial bioptome. $f p n$ Heart $\mathcal{1}$ 1972; 13: 464-9.

9 Bashour TT, Banks T, Cheng TO. Retrieval of lost catheters by a myocardial biopsy catheter device. Chest 1974; 66: 395-6.

10 Müller US, Gräwe G, Bender F. Träsvenöse Bergung zentral embolisierter Venenkatheterfragmente. $Z$ Kardiol 1979; 68: 180-2.

Requests for reprints to Dr T R D Shaw, Department of Cardiology, Western General Hospital, Edinburgh EH4 2XU. 